\title{
Vinpocetina de liberación prolongada: un posible tratamiento adyuvante en crisis epiléptica de inicio focal
}

\author{
Saúl Garza-Morales ${ }^{1 *}$, Eduardo Briceño-González², Hugo Ceja-Moreno³, José L. Ruiz-Sandoval³, \\ Fernando Góngora-Rivera ${ }^{4}$, Ildefonso Rodríguez-Leyva ${ }^{5}$ y Carlos G. Alonso-Rivera ${ }^{5}$ \\ ${ }^{1}$ Hospital Infantil de México Federico Gómez, Ciudad de México; ${ }^{2}$ Instituto Nacional de Neurología y Neurocirugía, Ciudad de México; ${ }^{3}$ Hospital \\ Civil de Guadalajara Fray Antonio Alcalde, Guadalajara, Jal.; ${ }^{4}$ Hospital Universitario Dr. José Eleuterio González, Universidad Autónoma de Nuevo \\ León, Monterrey, N.L.; ${ }^{5}$ Hospital Ángeles, San Luis Potosí, S. L.P. México
}

\begin{abstract}
Resumen
Introducción: La vinpocetina de liberación prolongada ha demostrado ser efectiva en el control de crisis de inicio focal en pacientes epilépticos con una baja frecuencia de eventos adversos. Se realizó un estudio clínico para evaluar la eficacia y tolerabilidad de la vinpocetina como tratamiento adyuvante en pacientes con este padecimiento. Métodos: Se realizó un estudio clínico, doble ciego, de grupos paralelos. Se reclutaron 87 pacientes con diagnóstico de epilepsia focal tratados con uno a tres fármacos antiepilépticos. Los pacientes se aleatorizaron para ser tratados con vinpocetina $(n=41)$ o placebo $(n=46)$ de manera adyuvante a su tratamiento, e ingresaron a la fase basal (4 semanas), a la fase de titulación (4 semanas) y a la fase de evaluación (8 semanas) conservando estables las dosis de la vinpocetina y de los fármacos antiepilépticos. Resultados: La vinpocetina fue más efectiva que el placebo en la reducción de las crisis al finalizar la fase de evaluación ( $p$ < 0.0001). El 69\% de los pacientes tratados con vinpocetina presentaron una reducción mayor al $50 \%$ en las crisis en comparación con el $13 \%$ de los pacientes tratados con placebo. No se presentaron diferencias significativas en cuanto a la presencia de efectos adversos en los pacientes tratados con vinpocetina comparados con los tratados con placebo. Los eventos adversos más frecuentes observados con vinpocetina fueron cefalea (7.9\%) y diplopía (5.2\%). Conclusiones: Como tratamiento adyuvante, la vinpocetina (2 $\mathrm{mg} / \mathrm{kg} / \mathrm{dia}$ ) redujo eficazmente la frecuencia de crisis epilépticas y demostró ser bien tolerada. Presenta un amplio perfil de seguridad y eventos adversos conocidos, que son transitorios y sin secuelas.
\end{abstract}

Palabras clave: Vinpocetina. Epilepsia. Crisis epilépticas. Fármacos antiepilépticos.

\section{Extended-release vinpocetine: a possible adjuvant treatment for focal onset epileptic seizures}

\begin{abstract}
Background: Extended-release vinpocetine is effective to control focal onset epileptic seizures with a low rate of adverse events. A clinical study was performed to evaluate the efficacy and tolerability of vinpocetine as an adjuvant treatment in patients with this condition. Methods: A double-blind clinical study of parallel groups was conducted, in which 87 patients
\end{abstract}

Ccorrespondencia:

*Saúl Garza Morales

E-mail: drsaulgarza@aol.com
Disponible en internet: 20-09-2019 Bol Med Hosp Infant Mex. 2019;76:215-224 www.bmhim.com 
with a diagnosis of focal epilepsy treated with one to three antiepileptic drugs were recruited. Patients were randomized to receive vinpocetine $(n=41)$ or placebo $(n=46)$ adjuvant to their treatment. Patients entered the baseline phase (4 weeks), the titration phase (4 weeks) and the evaluation phase (8 weeks), maintaining stable doses of vinpocetine and their respective antiepileptic drug treatment. Results: Vinpocetine was more effective than placebo in reducing seizures at the end of the evaluation phase $(p<0.0001)$. Sixty-nine percent of the vinpocetine-treated patients had a $50 \%$ reduction in seizures compared to $13 \%$ of placebo-treated patients. No significant differences in the presence of adverse effects in patients treated with vinpocetine compared to those treated with placebo were observed. The most frequent adverse events observed with vinpocetine were headache (7.9\%) and diplopia (5.2\%). Conclusions: As an adjuvant treatment, vinpocetine (2 mg/kg/day) effectively reduced the frequency of epileptic seizures and proved to be well tolerated. Vinpocetine has a wide safety profile and well-known adverse events, which are transient and with no sequelae.

Key words: Vinpocetine. Epilepsy. Epileptic seizure. Antiepileptic drugs.

\section{Introducción}

La epilepsia es un padecimiento crónico de etiología diversa que afecta del 1 al $2 \%$ de la población general ${ }^{1}$. En México, representa una afectación aproximada de 18 pacientes por cada 1,000 personas. Se manifiesta con cambios en la actividad motora, autonómica, sensitiva o de consciencia con carácter repetitivo y patrón estereotipado, secundaria a una descarga excesiva de un grupo de neuronas cerebrales, y puede estar asociada con manifestaciones paraclínicas ${ }^{1,2}$.

Estos fenómenos epilépticos se han clasificado de acuerdo con la correlación clínico-electroencefalográfica en dos grandes grupos: crisis parciales y crisis generalizadas, de acuerdo con la clasificación de la Liga Internacional Contra la Epilepsia (ILAE). En las crisis generalizadas, la descarga inicia simultáneamente en ambos hemisferios cerebrales. Muchas de las epilepsias generalizadas tienen un componente genético definido, con una función neurológica normal en la mayoría de los casos. En las epilepsias parciales, la crisis se origina en uno o múltiples focos localizados; sin embargo, se pueden propagar a todo el cerebro. La mayoría de las epilepsias parciales resultan de una afección del sistema nervioso central, aun cuando en muchos casos la naturaleza de la lesión nunca se identifica ${ }^{3}$.

La epilepsia se trata generalmente con bloqueadores de los canales de sodio sensibles a voltaje, como la carbamazepina, el ácido valproico y la fenitoína ${ }^{4,5}$; $\sin$ embargo, estos fármacos antiepilépticos (FAE) están asociados con diversos efectos adversos, interacciones medicamentosas y variaciones farmacocinéticas. Los FAE estándar producen, además, otros efectos indeseados a dosis terapéuticas, entre los cuales se encuentran el deterioro cognitivo y trastornos de la memoria ${ }^{6-9}$, que resultan particularmente preocupantes, sobre todo en niños en edad escolar que padecen epilepsia. Aproximadamente el $30-40 \%$ de los individuos con epilepsia presentan crisis a pesar de estar tomando monoterapia con FAE, por lo que se recurre a la politerapia con algún otro $F A E^{10}$.

Las fallas ocasionales en el tratamiento farmacológico y la presencia de diversos efectos adversos han llevado a probar nuevos tratamientos o esquemas de tratamiento. Uno de estos tratamientos es la vinpocetina, un alcaloide derivado de la vinca que actúa como bloqueador de los canales de sodio ${ }^{11}$. En estudios en cobayos, este fármaco ha demostrado que, al ser administrado cuatro horas antes de la inyección del agente convulsivante pentilentetrazol a una dosis de $2 \mathrm{mg} / \mathrm{kg}$, inhibe la aparición de convulsiones e impide la aparición de la actividad epiléptica cortical en el electroencefalograma durante los periodos ictal y postictal ${ }^{12}$. Dutov, et al. ${ }^{13}$ demostraron la eficacia del tratamiento con vinpocetina. En pacientes con epilepsia, sustituyeron un FAE del esquema múltiple de tratamiento por la vinpocetina, y encontraron que esta potencia la acción de otros FAE. Particularmente, esto se observó para los casos de convulsiones tónico-clónicas generalizadas. En un estudio posterior, Dutov, et al. ${ }^{14}$ demostraron que, en bebés hospitalizados por trauma intracraneal agudo ocurrido en el parto, el efecto adyuvante de la vinpocetina junto con el tratamiento convencional disminuyó la actividad epiléptica en comparación con los bebés que recibieron el tratamiento convencional.

Con base en los resultados observados de los efectos de la vinpocetina como antiepiléptico en modelos experimentales (realizados por el Dr. Nekrassov y la Dra. Sitges en el Instituto de Investigaciones Biomédicas, Universidad Nacional Autónoma de México), se decidió realizar un estudio piloto en pacientes con epilepsia parcial, quienes recibieron vinpocetina más carbamazepina o vinpocetina más valproato de magnesio; en este último grupo se observó mejoría, al igual que en los grupos de carbamazepina más valproato de 
magnesio. Cabe mencionar que en este estudio piloto no se observaron eventos adversos secundarios al tratamiento con vinpocetina. (Datos no publicados).

\section{Métodos}

\section{Diseño del estudio}

Se realizó un estudio clínico doble ciego, multicéntrico y longitudinal de grupos paralelos, utilizando un grupo control tratado con placebo. Los centros participantes fueron el Hospital Infantil de México Federico Gómez, el Instituto Nacional de Neurología y Neurocirugía, el Hospital Central de San Luis Potosí, el Hospital Universitario de Monterrey y el Hospital Civil de Guadalajara Fray Antonio Alcalde.

\section{Pacientes}

Se incluyeron 87 participantes con un rango de edad de entre 6 y 65 años (31 niños y 56 adultos), con un diagnóstico establecido de epilepsia con crisis focales con 0 sin generalización secundaria por, al menos, 24 semanas antes del reclutamiento. El diagnóstico de epilepsia focal fue establecido de acuerdo con los criterios de la ILAE y con un electroencefalograma compatible. Los pacientes debían presentar un mínimo de cuatro crisis al mes, corroboradas en el periodo basal, con por lo menos una crisis focal durante las ocho semanas previas al reclutamiento, y debieron ser tratados, por lo menos, con un FAE durante cuatro semanas previas al ingreso al estudio.

Los pacientes incluidos fueron aquellos tratados con carbamazepina, valproato de magnesio, topiramato 0 lamotrigina en monoterapia o cualquier combinación de estos.

Para poder participar en el estudio, las mujeres debían emplear un método anticonceptivo aceptado médicamente o no tener posibilidad de quedar embarazadas.

Se excluyeron del estudio pacientes con presencia de crisis generalizadas primarias, estado epiléptico en los 24 meses previos al ingreso al estudio, presencia de episodios epilépticos en salvas, desviaciones significativas de los valores de referencia por edad de los exámenes de laboratorio basales y con enfermedades progresivas o degenerativas (neurológicas o de otra índole), incluyendo padecimientos psiquiátricos que pudieran afectar la percepción o el registro de los eventos epilépticos.

Los 87 pacientes fueron aleatorizados utilizando una tabla de números aleatorios generada previamente al estudio: 46 pacientes fueron asignados al grupo placebo (además de su tratamiento con uno o varios FAE, recibieron tratamiento con placebo). Por otro lado, 41 pacientes fueron asignados al grupo vinpocetina (además de su tratamiento con FAE, se trataron con vinpocetina de liberación prolongada).

\section{Fases del estudio}

- Fase basal. A cada paciente se le realizó una biometría hemática, pruebas de función hepática, química sanguínea, electrolitos séricos, tiempo de protrombina, tiempo parcial de tromboplastina y niveles séricos de los FAE con los cuales estaban siendo tratados al momento de su inclusión al estudio. Durante cuatro semanas, se llevó un control estricto del número de crisis mediante el uso de un diario del paciente para corroborar el número de crisis epilépticas en el periodo. Durante esta fase, el régimen de FAE no fue modificado.

- Fase de titulación. Posteriormente, 41 pacientes fueron aleatorizados y tratados con $1 \mathrm{mg} / \mathrm{kg} / \mathrm{día}$ de vinpocetina como adyuvante de su tratamiento con FAE durante cuatro semanas. Se asignaron 46 participantes al grupo placebo, los cuales recibieron tratamiento con placebo sumado a su tratamiento con FAE. Los pacientes del grupo de vinpocetina que presentaron un control inadecuado de las crisis epilépticas (sin reducción $>50 \%$ respecto a la basal) recibieron un incremento en la dosis a $2 \mathrm{mg} / \mathrm{kg} / \mathrm{día}$ para la siguiente fase del estudio.

- Fase de evaluación. Durante cuatro semanas se mantuvo el régimen de tratamiento con FAE y con la dosis estandarizada de vinpocetina o placebo en la fase de titulación; se realizaron de nueva cuenta los estudios de laboratorio de la fase basal.

Después del término de cada fase, cada uno de los participantes acudió a una visita de control para la obtención de la información recabada en los diarios.

\section{Análisis estadístico}

El análisis estadístico fue realizado con el paquete estadístico $\mathrm{R}$ versión 3.2.2 de la siguiente manera:

\section{Porcentaje de Reducción de CRISIS}

- Análisis univariado de eficacia. Se realizó una prueba de normalidad de Shapiro-Wilk ${ }^{15}$ para conocer la distribución de la población, y posteriormente se aplicó una prueba rango-suma de Wilcoxon exacta para la 
diferencia de medianas ${ }^{16}$ y se construyó un intervalo de confianza del $97.5 \%$ de una sola cola.

- Análisis de eficacia por variables de confusión. Se ajustó un modelo logístico cuantílico regularizado ${ }^{17}$, seguido de la transformación logística de Bottai ${ }^{18}$ con intervalos de probabilidad del $95 \%$.

\section{Porcentaje de pacientes con decrementos PORCENTUALES DE AL MENOS $50 \%$}

- Análisis univariado de eficacia. Se utilizaron pruebas exactas de Fisher ${ }^{19}$ para las tablas de contingencia, y se construyeron intervalos de confianza del $97.5 \%$ de una sola cola.

- Análisis de eficacia por variables de confusión. Se ajustó un modelo logístico regularizado ${ }^{20,21}$ con intervalos de probabilidad del $95 \%$.

\section{EVENTOS ADVERSOS}

Las variables de seguridad y tolerabilidad incluyeron el porcentaje de pacientes con eventos adversos emergentes durante el estudio, sin importar la causa de estos ni el porcentaje de pacientes que abandonaron prematuramente el estudio debido a eventos adversos y la incidencia de eventos adversos serios.

\section{Resultados}

\section{Características demográficas y clínicas}

Las características demográficas y clínicas basales de los pacientes fueron comparables entre ambos grupos (Tabla 1). En los 56 pacientes adultos ( $\geq 18 \mathrm{y} \leq 65$ años), el diagnóstico predominante fue el de crisis parciales complejas, con una frecuencia del $88 \%$ de los casos (49 pacientes), seguido por crisis parciales simples, con el $9 \%$ (5 pacientes), y crisis parciales secundariamente generalizadas, con el $3 \%$ de los casos (2 pacientes). De los 31 niños (< 18 años), el 65\% (20 pacientes) presentaron crisis parciales complejas, el $19 \%$ (6 pacientes), crisis parciales secundariamente generalizadas, y el 16\% (5 pacientes), crisis parciales simples.

El tratamiento predominante utilizado como monoterapia en el grupo vinpocetina fue el valproato de magnesio, con el $39 \%$ (16 pacientes), y en el grupo placebo fue la carbamazepina, con el 33\% (15 pacientes). En la terapia combinada con dos fármacos predominó el tratamiento con valproato de magnesio y carbamazepina: el 58\% (24 pacientes) en el grupo vinpocetina y
Tabla 1. Características demográficas y clínicas basales de los pacientes en ambos grupos de tratamiento (niños y adultos)

\begin{tabular}{|l|c|c|}
\hline & $\begin{array}{c}\text { Vinpocetina } \\
(\mathbf{n}=\mathbf{4 1})\end{array}$ & $\begin{array}{c}\text { Placebo } \\
(\mathbf{n}=\mathbf{4 6})\end{array}$ \\
\hline Edad (años), media $\pm \mathrm{DE}$ & $22 \pm 12.06$ & $26 \pm 13.65$ \\
\hline Rango de edad (años) & $6-48$ & $7-60$ \\
\hline Sexo femenino, $\mathrm{n}(\%)$ & $23(56 \%)$ & $25(54 \%)$ \\
\hline Sexo masculino, n (\%) & $18(44 \%)$ & $21(46 \%)$ \\
\hline Peso (kg), media \pm DE & $54.3 \pm 21.32$ & $56.2 \pm 16.82$ \\
\hline IMC (kg/m²), media \pm DE & $23.06 \pm 6.28$ & $24.33 \pm 5.24$ \\
\hline \multicolumn{1}{|c|}{ Tratamiento basal, $\mathbf{n}(\%)$} & \\
\hline 1 FAE & $18(44)$ & $15(33)$ \\
\hline 2 FAE & $22(54)$ & $28(61)$ \\
\hline 3 FAE & $1(2)$ & $2(4)$ \\
\hline 4 FAE & 0 & $1(2)$ \\
\hline Número de crisis por & 7 & 8 \\
\hline mes (mediana) & & \\
\hline
\end{tabular}

FAE: fármacos antiepilépticos; DE: desviación estándar; IMC: índice de masa corporal.

el $60 \%$ (28 pacientes) en el grupo placebo. No se encontraron diferencias significativas entre las dosis de antiepilépticos al comparar entre el grupo vinpocetina y el grupo placebo. El uso de benzodiacepinas de rescate fue similar entre ambos grupos.

\section{Porcentaje de reducción de crisis}

\section{ANÁlISIS UNIVARIADO DE EFICACIA}

En la figura 1, se comparan los cuantiles del grupo vinpocetina y placebo de las distribuciones de los porcentajes de reducción de las crisis por grupo con los cuantiles de una distribución normal. Al observarse importantes desviaciones en la normalidad, se optó por realizar la prueba de Shapiro-Wilk, que mostró que las poblaciones de los grupos de vinpocetina y placebo no siguieron una distribución normal (valor estadístico para vinpocetina: $0.74 ; p<0.001$; para placebo: 0.81 ; $p<0.001$.

Considerando que los porcentajes de reducción de crisis no siguen una distribución normal, el análisis estadístico se enfocó en la diferencia de medianas. La prueba de rango-suma de Wilcoxon exacta con un tamaño de prueba de 0.025 para la diferencia de 

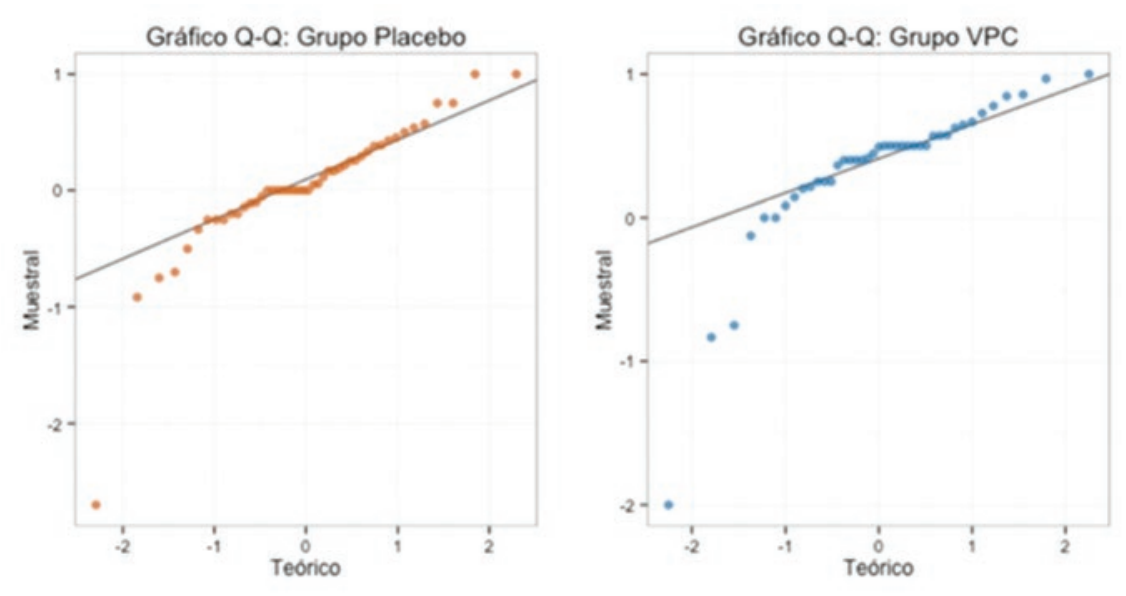

Figura 1. Gráficos Q-0 del porcentaje de reducción de crisis por grupo.

VPC: Vinpocetina.

medianas arrojó una estadística $(\mathrm{W}=1,391 ; \mathrm{p}<0.001)$, lo cual indica que el porcentaje de reducción de crisis al finalizar las 12 semanas de tratamiento (fase basal + fase de titulación + fase de evaluación) fue mayor para el grupo de vinpocetina, comparado con el grupo que recibió placebo.

\section{ANÁlISIS dE EFICACIA POR VARIABLES DE CONFUSIÓN}

Se emplearon modelos logísticos regularizados que permitieron analizar el posible efecto de variables confusoras, para lo cual se utilizó un modelo logístico cuantílico, con intervalos de probabilidad del 95\%. Las variables explicativas incluidas fueron el tratamiento (placebo o vinpocetina), sexo, edad (adultos o niños), índice de masa corporal (IMC) y número de crisis (Tabla 2).

- Tratamiento. La mediana estimada para el porcentaje de reducción de crisis para el placebo fue del $0 \%$ (intervalo de -20-25\%) y para el grupo de vinpocetina, del $45 \%$ (intervalo de $25-60 \%$ ). Esto indicó que el porcentaje de reducción de crisis fue mayor en el grupo de vinpocetina (Fig. 2).

- Sexo. Se evaluó si el efecto del tratamiento con vinpocetina era diferente entre el sexo masculino y el sexo femenino. Se observó una mayor reducción en el porcentaje de crisis en el grupo de vinpocetina en ambas poblaciones. Sin embargo, al comparar entre los sexos, se aprecia que el efecto del tratamiento no varió (Fig. 3A).

- Edad. Se comparó el efecto del tratamiento con vinpocetina en niños (< 18 años) y adultos $(\geq 18$
Tabla 2. Mediana del decremento porcentual de los grupos por características de los pacientes, por grupo y global

\begin{tabular}{|l|c|c|c|}
\hline \multirow{2}{*}{} & \multicolumn{3}{|c|}{$\begin{array}{c}|c| \\
\text { Mediana crisis }\end{array}$} \\
\cline { 2 - 4 } & Placebo & Vinpocetina & Global \\
\hline $\begin{array}{l}\text { Sexo } \\
\text { Femenino } \\
\text { Masculino }\end{array}$ & 0 & 35 & 25 \\
\hline Edad & 6 & 50 & 26 \\
\hline Adultos & & & \\
Niños & 0 & 40 & 21 \\
\hline Número basal de crisis & 11 & 50 & 29 \\
\hline 4 a 6 & & & \\
7 a 11 & 0 & 40 & 25 \\
\hline 12 a 174 & 14 & 47 & 0 \\
\hline IMC & & 57 & 25 \\
\hline 20 & 11 & & \\
\hline $20-25$ & 0 & 50 & 35 \\
\hline 25 & 0 & 40 & 28 \\
\hline
\end{tabular}

IMC: índice de masa corporal.

años). El análisis demostró una mayor reducción en el porcentaje de crisis con el tratamiento con vinpocetina. El efecto del tratamiento no mostró diferencias significativas comparando ambos grupos de edad. Asimismo, se observó una mayor dispersión de las estimaciones para el grupo de $<18$ años (Fig. 3B).

- Número basal de crisis. El número de crisis basales se dividió, de manera que una tercera parte de la muestra se ubicara en cada nivel $(4-6,7-11,12+$ 


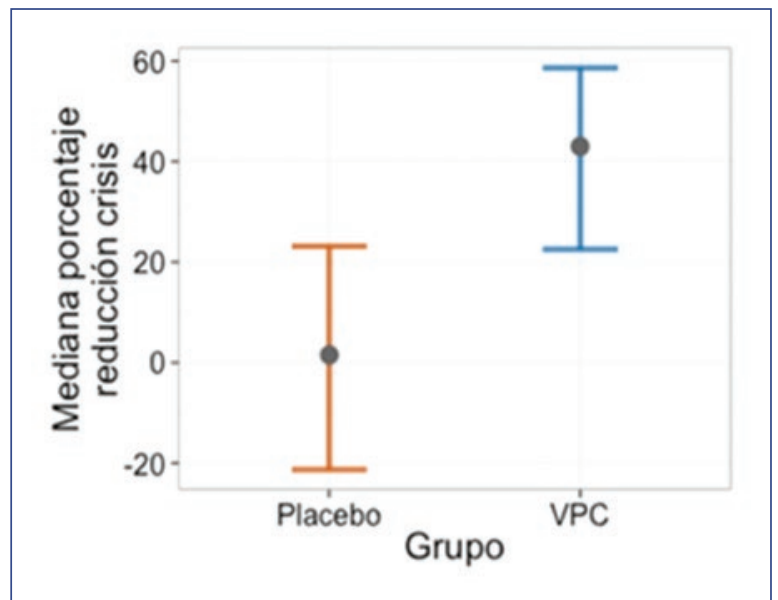

Figura 2. Medianas del decremento porcentual de crisis por grupo estimadas por el modelo con intervalos de probabilidad de $95 \%$.

VPC: Vinpocetina.

crisis). El porcentaje de reducción de crisis fue mayor para el grupo de vinpocetina en todos los niveles, $y$ se observó que los límites inferiores de probabilidad fueron mayores para el nivel de 12+ crisis (Fig. 4).

- IMC. Se observó un mayor porcentaje de reducción de crisis en el grupo de vinpocetina, sin importar el IMC $(<20,20-25,>25)$. No se obtuvo evidencia de que el IMC influyera en la respuesta del grupo de vinpocetina en comparación con el grupo placebo.

\section{Porcentaje de pacientes con decrementos porcentuales del $50 \%$}

\section{ANÁLISIS UNIVARIADO DE EFICACIA}

La proporción de pacientes respondedores al 50\% en el grupo de vinpocetina fue del $49 \%$, mientras que en el grupo de placebo solamente fue del $15 \%$. También se realizó una prueba exacta de Fisher entre ambos grupos de tratamiento para un tamaño de muestra de 0.025 , obteniéndose una razón de momios de 5.2 $y$ un valor de $p$ de 0.00007 , con lo cual se obtuvo una diferencia estadísticamente significativa para los pacientes tratados con vinpocetina.

\section{ANÁLISIS DE EFICACIA POR VARIABLES dE CONFUSIÓN}

Se empleó un modelo logístico regularizado que estima el logit de la probabilidad de que los porcentajes de reducción de crisis sean $\geq 50 \%$. Las variables explicativas incluidas fueron el tratamiento (placebo 0 vinpocetina), sexo, edad (adultos o niños), IMC y número de crisis (Tabla 2).

- Tratamiento. La probabilidad estimada de un decremento porcentual de crisis del $50 \%$ fue de 0.5 para el grupo de vinpocetina (intervalo 0.4 a 0.7 ) y de 0.25 para el grupo de placebo (intervalo de 0.10 a 0.40 ), lo cual señala que la probabilidad de que las crisis se reduzcan $\geq 50 \%$ en el grupo de vinpocetina es mayor (Fig. 5).

- Sexo. Se evaluó el efecto del tratamiento con vinpocetina entre el sexo masculino y el sexo femenino. En ambos, el grupo de vinpocetina mostró mayor porcentaje de pacientes con decrementos porcentuales de al menos el 50\%. Sin embargo, al comparar entre los sexos, se aprecia que el efecto del tratamiento no varió (Fig. 6A).

- Edad. Se comparó el efecto del tratamiento con vinpocetina en niños ( $<18$ años) y adultos ( $\geq 18$ años). El análisis mostró que el tratamiento con vinpocetina tiene mayor porcentaje de pacientes con decrementos porcentuales de al menos el $50 \%$. El efecto del tratamiento no varía de manera significativa comparando ambos grupos de edad. Se observó una mayor dispersión de las estimaciones para el grupo de niños (Fig. 6B).

- Número basal de crisis. El número de crisis basales se dividió, de manera que una tercera parte de la muestra se ubicara en cada nivel $(4-6,7-11,12+$ crisis). El porcentaje de pacientes con decrementos porcentuales de al menos el $50 \%$ fue mayor para el grupo de vinpocetina en todos los niveles, y se observó que los límites inferiores de probabilidad son mayores para el nivel de 12+ crisis (Fig. 7).

- IMC. El grupo de vinpocetina mostró un mayor porcentaje de reducción de crisis, sin importar el IMC $(<20,20-25,>25)$. No se obtuvo evidencia de que el IMC afecte la respuesta del grupo de vinpocetina en comparación con el grupo placebo.

\section{Resultados de laboratorio}

Los resultados de laboratorio no mostraron diferencias estadísticamente significativas en ninguno de los grupos de tratamiento para ninguna de las variables evaluadas. Los cambios individuales en los valores de laboratorio para ambos grupos se mantuvieron dentro de los rangos de normalidad establecidos por edad del paciente.

\section{Eventos adversos}

Los eventos adversos en su mayoría fueron leves y conocidos, con predominio de cefalea (7.89\%). Sin 


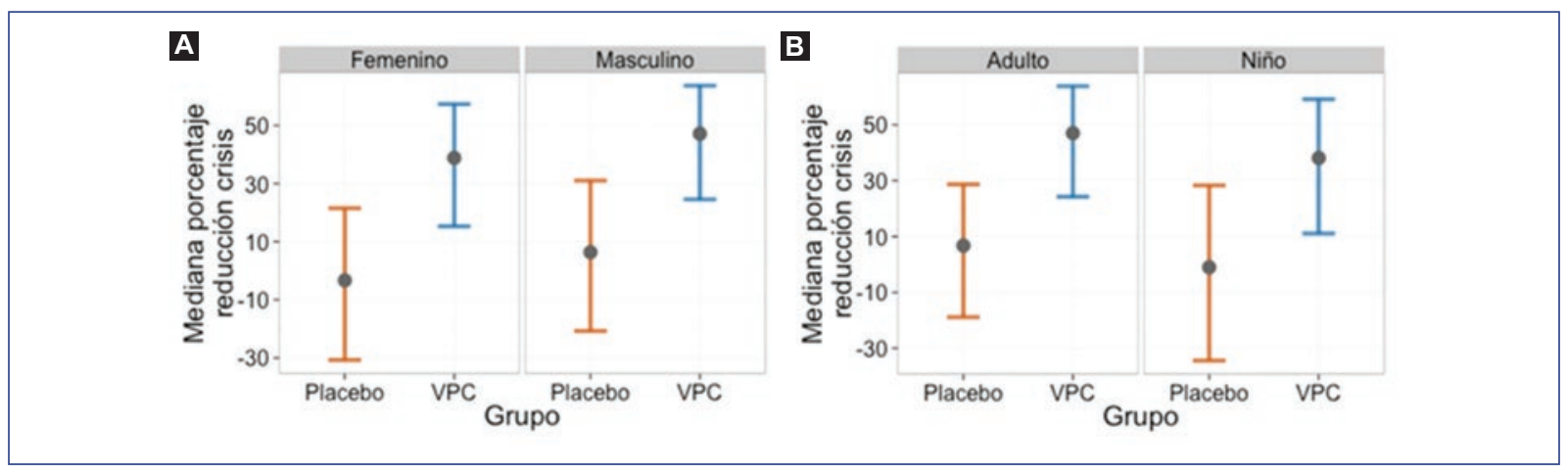

Figura 3. A: Medianas del decremento porcentual de crisis por grupo y por sexo estimadas por el modelo con intervalos de probabilidad de $95 \%$. B: Medianas del decremento porcentual de crisis por grupo y por grupo de edad estimadas por el modelo con intervalos de probabilidad de $95 \%$.

VPC: Vinpocetina.

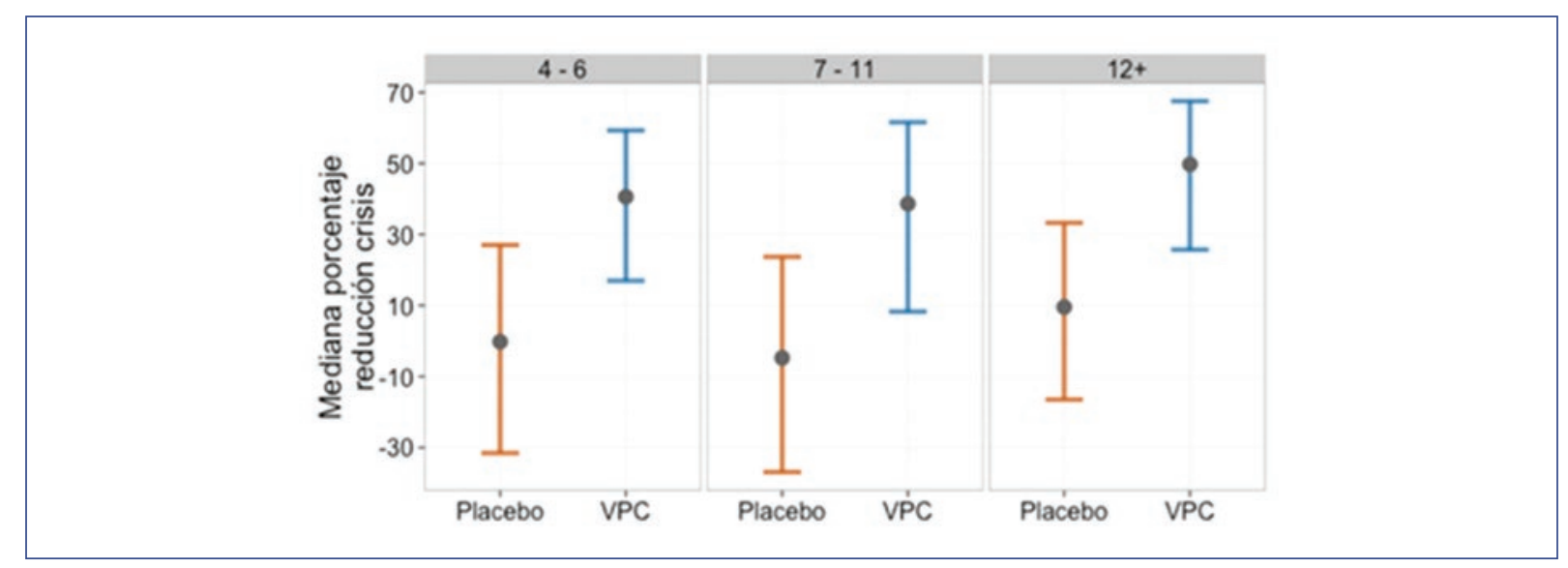

Figura 4. Medianas del decremento porcentual de crisis por grupo y por nivel basal de crisis estimadas por el modelo con intervalos de probabilidad de $95 \%$.

VPC: Vinpocetina.

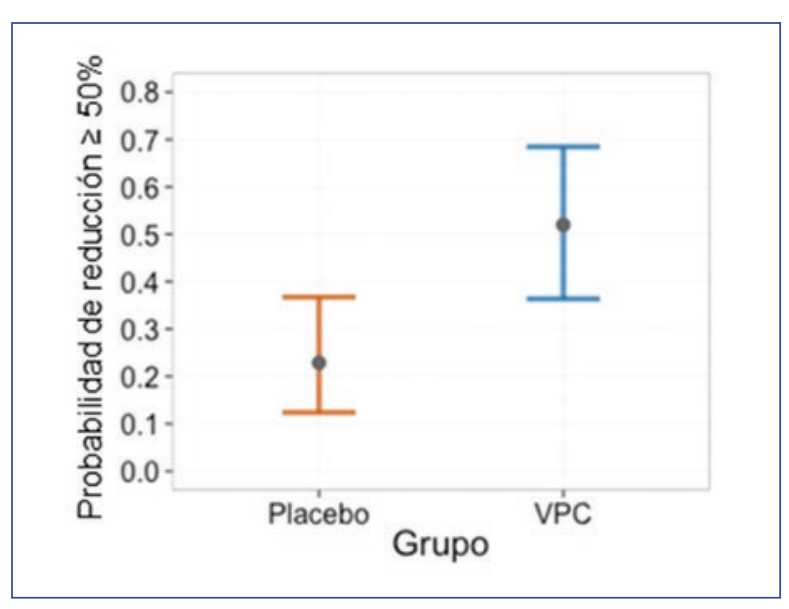

Figura 5. Medianas del decremento porcentual de crisis por grupo y por índice de masa corporal estimadas por el modelo con intervalos de probabilidad de $95 \%$. VPC: Vinpocetina. embargo, al interrogar nuevamente a los pacientes ( $>18$ años de edad), la cefalea fue parte de la sintomatología postictal habitualmente presentada. Dos eventos adversos ocasionaron baja del protocolo y suspensión de vinpocetina (5.2\%): un paciente presentó vómito, y otro mostró falla terapéutica a la fórmula, e incluso descontrol de las crisis (ambos pacientes pediátricos). Otros eventos adversos reportados en el grupo vinpocetina fueron mareo, diplopía, polidipsia y poliuria. En el grupo placebo se reportaron únicamente mareo y cefalea (Tabla 3 ).

\section{Discusión}

Este estudio doble ciego, aleatorizado, de grupos paralelos abordó, de manera novedosa, la administración de la vinpocetina como adyuvante en la terapia 


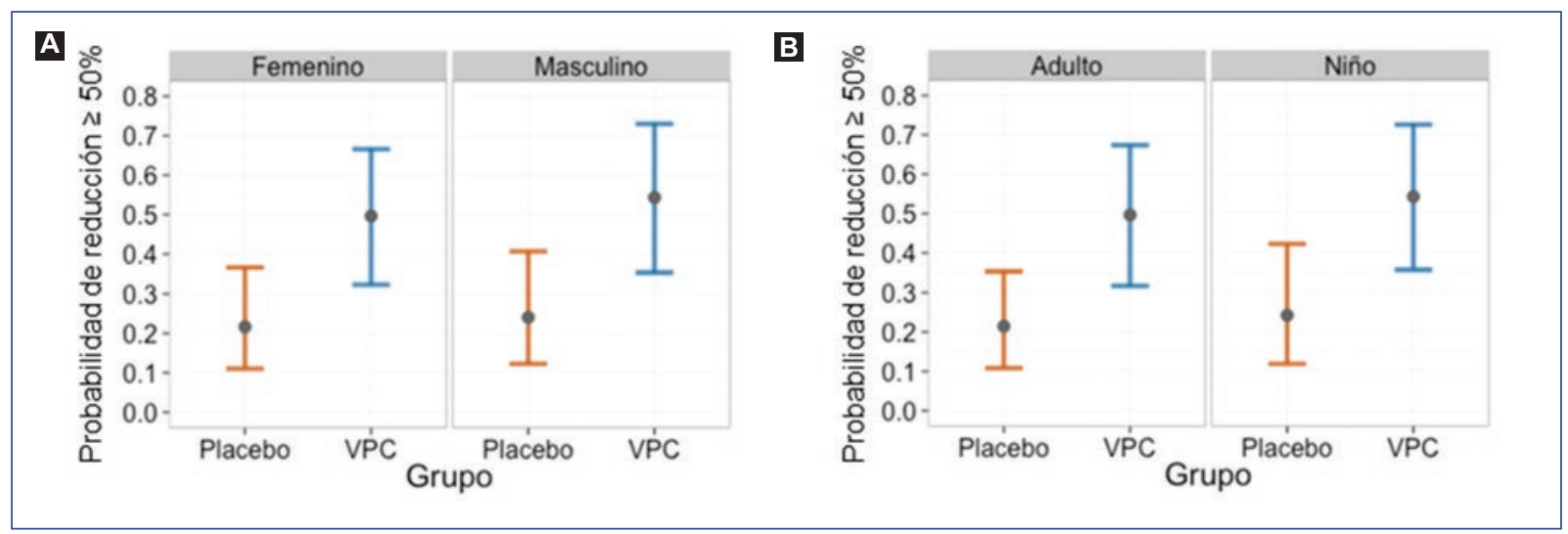

Figura 6. A: Probabilidades de ser respondedor al 50\% por grupo estimadas por el modelo con intervalos de probabilidad de 95\%. B: Probabilidades de ser respondedor al 50\% por grupo y por sexo estimadas por el modelo con intervalos de probabilidad de $95 \%$.

VPC: Vinpocetina.

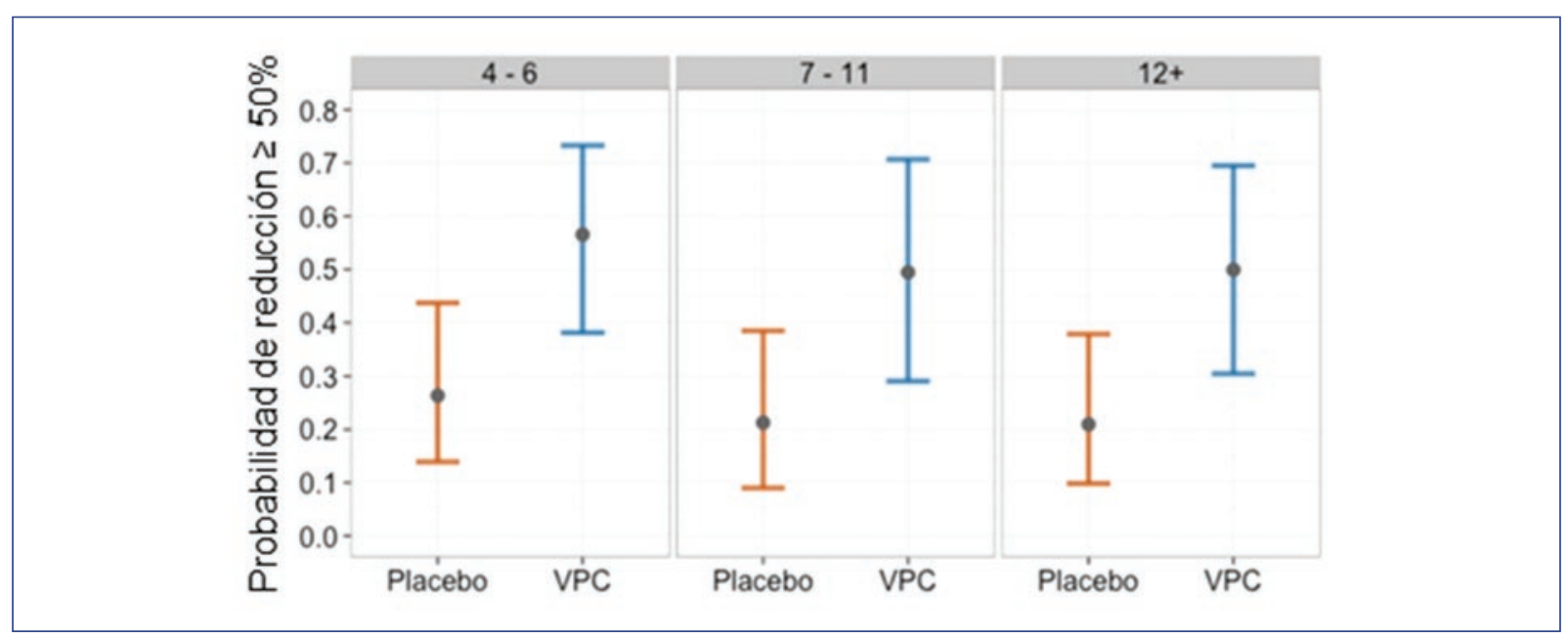

Figura 7. Probabilidades de ser respondedor al $50 \%$ por grupo y por edad estimadas por el modelo con intervalos de probabilidad de $95 \%$.

VPC: Vinpocetina.

para el tratamiento de la epilepsia en pacientes con descontrol de crisis, a pesar de un uso apropiado de $\mathrm{FAE}$, y demostró, por medio de un análisis univariado de eficacia, que la terapia conjunta de FAE con vinpocetina reduce la aparición de crisis epilépticas en pacientes pediátricos y adultos y que esta respuesta se puede encontrar en al menos el $50 \%$ de los pacientes tratados con vinpocetina. Este estudio refuerza los resultados presentados por Dutov, et al., ya que se utilizó la vinpocetina como tratamiento adyuvante de las crisis epilépticas y, a diferencia del artículo mencionado, se incluyó una clasificación para la epilepsia y un tamaño de muestra mayor ${ }^{13,14}$. El análisis de eficacia por variables de confusión demostró que los resultados obtenidos no varían con respecto al sexo, la edad, el número basal de crisis y el IMC. Las dispersiones mayores en los pacientes pediátricos, que se aprecian tanto en el porcentaje de reducción de crisis como en el porcentaje de pacientes con decrementos porcentuales al menos del $50 \%$, se deben a que hay un menor número de pacientes pediátricos, especialmente en el grupo tratado con placebo.

Además de los resultados obtenidos, el perfil de tolerabilidad y seguridad de la vinpocetina fue favorable. Los eventos reportados como graves se presentaron en la población pediátrica, de los cuales, uno se relacionó 
Tabla 3. Eventos adversos reportados durante el desarrollo del estudio

\begin{tabular}{|c|c|c|c|c|}
\hline Sistema de clasificación de órganos & \multicolumn{2}{|c|}{$\begin{array}{l}\text { Tratamiento base + vinpocetina } \\
\qquad(\mathrm{n}=41)\end{array}$} & \multicolumn{2}{|c|}{$\begin{array}{l}\text { Tratamiento base + placebo } \\
\qquad(n=46)\end{array}$} \\
\hline \multirow[t]{2}{*}{ Trastornos del sistema nervioso } & Mareo & $2(4.8 \%)$ & Mareo & $3(6.5 \%)$ \\
\hline & Cefalea & $2(4.8 \%)$ & Alteraciones del sueño & $1(2.1 \%)$ \\
\hline Trastornos oculares & Diplopía & $1(2.4 \%)$ & Diplopía & $1(2.1 \%)$ \\
\hline \multirow[t]{3}{*}{ Trastornos gastrointestinales } & \multirow{3}{*}{$\begin{array}{l}\text { Vómito (reportado como } \\
\text { evento adverso grave) }\end{array}$} & \multirow[t]{3}{*}{$1(2.4 \%)$} & Vómito & $1(2.1 \%)$ \\
\hline & & & Náuseas & $1(2.1 \%)$ \\
\hline & & & Dolor epigástrico & $1(2.1 \%)$ \\
\hline Trastorno del sistema respiratorio & \multicolumn{2}{|l|}{ - } & Rinorrea & $1(2.1 \%)$ \\
\hline Trastornos urinarios & Poliuria & $1(2.4 \%)$ & \multicolumn{2}{|l|}{ - } \\
\hline Trastorno del sistema endocrino & Polidipsia & $1(2.4 \%)$ & \multicolumn{2}{|l|}{ - } \\
\hline
\end{tabular}

con falla terapéutica, y el otro, con vómito incontrolable. La falla terapéutica es una realidad en pacientes epilépticos y guarda relación con diversos factores, incluso del tipo epigenético. Por otro lado, el paciente con vómito presentó un proceso gastrointestinal de posible origen infeccioso. Los eventos adversos más frecuentes durante el estudio incluyeron cefalea y mareo, ambos similares a otros eventos adversos reportados por otros FAE con similar mecanismo de acción.

En modelos animales, la vinpocetina ha demostrado una mayor eficacia al disminuir la permeabilidad de canales de sodio y calcio, controlando la liberación de glutamato en las terminales nerviosas en comparación con otros FAE, incluyendo la carbamazepina, lamotrigina, ácido valproico y topiramato $22-24$. Esto explicaría la capacidad que la vinpocetina mostró para controlar la frecuencia de crisis epilépticas en los pacientes que participaron en el estudio.

Una de las principales limitaciones del presente estudio es el reclutamiento del $50 \%$ de los pacientes originalmente calculados para la muestra. Sin embargo, el uso de un análisis descriptivo, exploratorio, el análisis univariado de eficacia y el análisis de eficacia por variables de confusión indicaron que los resultados obtenidos no cambiarían con el incremento del tamaño de la muestra, y solamente aumentaría la precisión de estos. Por lo tanto, no fue necesario incrementar el número de pacientes y realizar un nuevo análisis al término del reclutamiento. Por otro lado, el periodo de seguimiento de los pacientes (12 semanas en total) es relativamente corto para un padecimiento crónico como la epilepsia, por lo que el perfil de eficacia y seguridad de la vinpocetina y su comportamiento en comparación con otros FAE se tendrán que evaluar en un periodo mayor en futuros estudios.

La vinpocetina de liberación prolongada ha demostrado ser un fármaco efectivo en el control de crisis de inicio focal en pacientes con epilepsia, con una baja frecuencia de eventos adversos. La liberación prolongada de este fármaco disminuye el número de tabletas administradas al paciente, lo que facilita la adherencia al tratamiento.

En conclusión, este estudio muestra el potencial terapéutico de la vinpocetina. Sin embargo, serán necesarios futuros estudios clínicos de acuerdo con las recomendaciones de la ILAE ya que, durante el proceso de aprobación de un FAE, se requiere aumentar la experiencia en terapia adyuvante y avanzar hacia la monoterapia, por lo que se plantea continuar con un protocolo de conversión en pacientes con epilepsia focal.

\section{Responsabilidades éticas}

Protección de personas y animales. Los autores declaran que para esta investigación no se han realizado experimentos en seres humanos ni en animales.

Confidencialidad de los datos. Los autores declaran que han seguido los protocolos de su centro de trabajo sobre la publicación de datos de pacientes.

Derecho a la privacidad y consentimiento informado. Los autores han obtenido el consentimiento informado de los pacientes o individuos referidos en el artículo. Este documento obra en poder del autor de correspondencia. 


\section{Conflicto de intereses}

Los autores declaran no tener ningún conflicto de intereses.

\section{Financiamiento}

El estudio se financió en su totalidad por un fondo de apoyo a la investigación de Psicofarma, S.A. de C.V.

\section{Agradecimientos}

A todos los pacientes que formaron parte del estudio y a los cinco investigadores mexicanos encargados de coordinar cada uno de los centros que participaron en el reclutamiento de pacientes.

\section{Bibliografía}

1. Fisher RS, Acevedo C, Arzimanoglou A, Bogacz A, Cross JH, Elger CE, et al. ILAE official report: a practical clinical definition of epilepsy. Epilepsia. 2014;55:475-82.

2. Epilepsiamexico.gob.mx [Internet]. México: PPE. Programa Prioritario de Epilepsia; 2011. Disponible en: http://www.epilepsiamexico.gob.mx/index.html.

3. Scheffer IE, French J, Hirsch E, Jain S, Mathern GW, Moshe SL, et al. Classification of the epilepsies: New concepts for discussion and debate-Special report of the ILAE Classification Task Force of the Commission for Classification and Terminology. Epilepsia Open. 2016;1:37-44.

4. Lingamaneni R, Hemmings HCJr. Differential interaction of anaesthetics and antiepileptic drugs with neuronal $\mathrm{Na}+$ channels, $\mathrm{Ca} 2+$ channels, and GABA(A) receptors. Br J Anaesth. 2003;90:199-211.

5. Ambrosio AF, Silva AP, Malva JO, Soares-da-Silva P, Carvalho AP, Carvalho CM. Inhibition of glutamate release by BIA 2-093 and BIA 2-024, two novel derivatives of carbamazepine, due to blockade of sodium but not calcium channels. Biochem Pharmacol. 2001;61:1271-5.
6. Vermeulen J, Aldenkamp AP. Cognitive side-effects of chronic antiepileptic drug treatment: a review of 25 years of research. Epilepsy Res. 1995;22:65-95.

7. Gates JR. Side effect profiles and behavioral consequences of antiepileptic medications. Epilepsy Behav. 2000;1:153-9.

8. Brunbech $L$, Sabers A. Effect of antiepileptic drugs on cognitive function in individuals with epilepsy: a comparative review of newer versus older agents. Drugs. 2002;62:593-604.

9. Schmidt $D$. The clinical impact of new antiepileptic drugs after a decade of use in epilepsy. Epilepsy Res. 2002;50:21-32.

10. Tatum WO 4th, Benbadis SR, Vale FL. The neurosurgical treatment of epilepsy. Arch Fam Med. 2000;9:1142-7.

11. Erdo SA, Molnár P, Lakcis V, Bence JZ, Tömösközi Z. Vincamine and vincanol are potent blockers of voltage-gated $\mathrm{Na}+$ channels. Eur J Pharmacol. 1996;314:69-73.

12. Nekrassov V, Sitges M. Vinpocetine inhibits the epileptic cortical activity and auditory alterations induced by pentylenetetrazole in the guinea pig in vivo. Epilepsy Res. 2004;60:63-71.

13. Dutov AA, Tolpyshev BA, Petrov AP, Gladun VN. [Use of cavinton in epilepsy]. Zh Nevropatol Psikhiatr Im S S Korsakova. 1986;86:850-5.

14. Dutov AA, Gal'tvanitsa GA, Volkova VA, Sukhanova ON, Lavrishcheva TG Petrov AP. [Cavinton in the prevention of the convulsive syndrome in children after birth injury]. Zh Nevropatol Psikhiatr Im S S Korsakova. 1991;91:21-2.

15. Shapiro SS, Wilk MB. An analysis of variance test for normality (complete samples). Biometrika. 1965;52:591-611.

16. Wilcoxon F. Individual comparisons by ranking methods. Biometr Bull. 1945;1:80-3.

17. $\mathrm{Yu} \mathrm{K}$, Moyeed RA. Bayesian quantile regression. Stat Probabil Lett. 2001;54:437-47.

18. Bottai M, Cai B, McKeown RE. Logistic quantile regression for bounded outcomes. Stat Med. 2010;29:309-17.

19. Fisher RA. The logic of inductive inference. J R Stat Soc. 1935;98:39-82.

20. Gelman A, Jakulin A, Pittau MG, Yu-Sung Su. A weakly informative default prior distribution for logistic and other regression models. Ann Appl Stat. 2008;2:1360-83.

21. Gelman A, Hill J. Data Analysis Using Regression and Multilevel/Hierarchical Models. New York: Cambridge University Press; 2007.

22. Galván $\mathrm{E}$, Sitges M. Characterization of the participation of sodium channels on the rise in $\mathrm{Na}+$ induced by 4-aminopyridine (4-AP) in synaptosomes. Neurochem Res. 2004;29:347-55.

23. Sitges M, Galván E, Nekrassov V. Vinpocetine blockade of sodium channels inhibits the rise in sodium and calcium induced by 4-aminopyridine in synaptosomes. Neurochem Int. 2005;46:533-40.

24. Sitges M, Chiu LM, Guarneros A, Nekrassov V. Effects of carbamazepine, phenytoin, lamotrigine, oxcarbazepine, topiramate and vinpocetine on $\mathrm{Na}+$ channel-mediated release of [3H]glutamate in hippocampal nerve endings. Neuropharmacology. 2007;52:598-605. 